\title{
Supercomputer Simulation of the Modes of Colliding Microdrops of Water*
}

\author{
by \\ Donald Greenspan and Larry F. Heath
}

TR \#273

September 1990

\begin{abstract}
Fluid microdrop collisions are important in both chemical and meteorological studies. In this paper, a three dimensional, molecular model of colliding microdrops of water is developed and explored. Fundamental modes for coherent collisions are described and exhibited, as are results for noncoherent collisions.
\end{abstract}

* Computations performed at the University of Texas Center for High Performance Computing. 


\section{Introduction}

Fluid microdrop collisions are important in both chemical and meteorological studies [1], [5], [6]. In this paper we develop a simple molecular model for studying related collision modes and, in particular, apply it to the study of water.

For $\mathrm{N}$ given water molecules $\mathrm{P}_{1}, \mathrm{P}_{2}, \ldots, \mathrm{P}_{\mathrm{N}}$, which interact in accordance with classical molecular mechanics, the motion of each $\mathrm{P}_{\mathrm{i}}$ can be approximated [2] from given initial data by solving the system of second order, nonlinear, ordinary differential equations.

$$
\frac{\mathrm{d}^{2} \overrightarrow{\mathrm{r}}_{i}}{\mathrm{dT}^{2}}=\sum_{\substack{\mathrm{j}=1 \\ \mathrm{j} \neq \mathrm{i}}}^{\mathrm{N}}\left(-\frac{16.5}{\mathrm{r}_{\mathrm{ij}}^{3}}+\frac{158.6}{\mathrm{r}_{\mathrm{ij}}^{5}}\right) \frac{\overrightarrow{\mathrm{r}}_{\mathrm{ji}}}{\mathrm{r}_{\mathrm{ij}}}, \quad \mathrm{i}=1,2, \ldots, \mathrm{N},
$$

in which $\vec{r}_{j}$ is the position vector of $P_{i}, \vec{r}_{j i}$ is the vector for $P_{j}$ to $P_{i}$ and $r_{i j}$ is the length of $\vec{r}_{\mathrm{ji}} \cdot$ Throughout, the unit of length is the angstrom, and $\mathrm{T}=10^{5.5} \mathrm{t}$ secs, $\mathrm{T}_{\mathrm{k}}=\mathrm{k} \Delta \mathrm{T}, \mathrm{k}=0$, $1,2, \cdots$.

In three dimensions, with $\mathrm{N}=4102$, which is the number of molecules to be considered, the system (1.1) consists of 12306 second order, nonlinear, ordinary differential equations in the position coordinates $\mathrm{x}, \mathrm{y}, \mathrm{z}$. The numerical solution [3] of this system will be generated on a CRAY X-MP/24 supercomputer with time step $\Delta \mathrm{T}=0.0002$.

\section{Single Drop Generation}

In order to study the modes of two colliding water drops, it is convenient first to generate a single water drop, which is done as follows. In the space cube $-31.0 \leq \mathrm{x} \leq 31.0$, 
$-31.0 \leq \mathrm{y} \leq 31.0,-31.0 \leq \mathrm{z} \leq 31.0$, molecules are placed at the grid points generated by the choices $\Delta x=\Delta y=\Delta z=3.1$. The grid size 3.1 $\AA$ is the one which makes the term within the parentheses of (1.1) equal to zero for $r=3.1$. Next, the molecules outside the sphere $x^{2}+y^{2}+z^{2}=26^{2}$ are deleted and each of the remaining molecules is assigned a random velocity [3] in the range $|v| \leq 0.02$. At initial time, then, there are 2517 molecules which are thereafter allowed to interact in accordance with (1.1) for 31000 time steps. At $T_{31000}$, all molecules whose position coordinates satisfy $r>26$ are deleted, reducing the number to 2051. The simulation is then continued to $T_{88000}$, but with all velocities damped by a reset to zero at $\mathrm{T}_{40500}, \mathrm{~T}_{48000}, \mathrm{~T}_{53000}, \mathrm{~T}_{58000}, \mathrm{~T}_{63000}, \mathrm{~T}_{68000}$, and $\mathrm{T}_{73000}$. At $\mathrm{T}_{78000}$ the velocities are damped by the factor 0.5 . The damping process so imposed cools the molecular configuration so that at $\mathrm{T}_{88000}$ the temperature [4] of the resulting drop is $45^{\circ} \mathrm{C}$. This drop is shown in Figure 1.

\section{Collision Modes}

In order to study collision modes, the single drop generated in Section 2 is duplicated by mirror imaging. The resulting two drops are set symmetrically $3 \AA$ apart about the YZ plane, as shown in Figure 2. To elucidate the motions of individual molecules during collision, the drops are displayed in different shadings. To avoid complete symmetry, the velocity of any molecule and that of its mirror image molecule are taken to be the same. In addition, the time counter is reset to zero.

To simulate collision, we will assume that each molecule of the light drop, on the left in Figure 2, has its velocity increased initially by $\overrightarrow{\mathbf{v}}^{*}$, while each molecule of the dark drop 
has its velocity decreased by $\vec{\nabla}^{*}$.

As a first case, let $\vec{v}^{*}=(0,0,0)$, so that the two drops are allowed to interact with no changes in velocity. Then, Figure 3 shows, at the indicated times, an oblate spheroid oscillation mode. After an extended period of time, the large boundary gradients due to surface tension transform this mode into a relatively spherical drop which exhibits small oscillations throughout its surface. Indeed, in this example and in all cohesive interactions to be described, the so-called oscillation modes [1], [5], [6] are, in reality, dynamical configurations which, in time, transform into a spherical configuration.

Next, set $\overrightarrow{\mathrm{v}}^{*}=(2.2,0.2,0.0)$. Figure 4 then shows at the indicated times the development of a raindrop mode [5].

Setting $\overrightarrow{\mathrm{v}}^{*}=(-2.0,4.5,0)$ yields, as shown in Figure 5, a dumbbell mode [1], [6].

Next, selecting the largest speed of any case yet considered by the choice $\vec{v}^{*}=(0.2$, 8.0, 0.0) yields, as shown in Figure 6, a noncohesive, brush type collision, in which each of the drops forms a tear drop mode [6].

Finally, increasing the speed still further, but in a fashion which results in more direct collision, the choice $\vec{v}^{*}=(5.0,10.0,0.0)$ yields, as shown in Figure 7 , a non-cohesive collision which exhibits an initial clean slicing effect, the molecular transfer during and after collision, and the loss of molecules after separation.

\section{4. $\quad$ Remarks}


Our vectorized computer code required 2000 seconds of CPU time for 1000 numerical time steps. Budgetary considerations then limited our simulations to only seventeen cases in addition to those described in Section 3. These are summarized as follows. Choices of $\vec{v}^{*}$ which resulted in high velocity, direct collisions, like $\vec{v}^{*}=(12.0,0.2$, 0.0), yielded explosive type reactions, as shown in Figure 8. Large momentum effects, as in the case $\vec{v}^{*}=(0.2,5.0,0.0)$, would often have dumbbell modes develop into a peanut shape in their transition to sphericity, as shown in Figure 9. And small perturbations of the parameters in the examples in Section 3 resulted in entirely similar results, confirming the physical stability of the modes.

Though our results indicated that cohesion is dependent upon both speed and angle of contact, we did not at present accumulate enough data to be able to deduce a quantitative relationship. This might have been possible if we had fixed the angle of contact to be $49^{\circ}$ and then varied the speed, as in [6], but we felt that the available computer time would be better used to show that the modes produced experimentally by others [1], [6] could be reproduced also theoretically, even though our model assumes interaction in a vacuum and our drops have diameters of only $20.7 \AA$.

Note, finally, that the computer programs for both the computations and the graphics are available [3]. 


\section{REFERENCES}

1. J. R. Adam, N. R. Lindblad, and C. D. Hendricks, "The collision, coalescence, and disruption of water droplets," J. Appl. Phys., 39, 1968, p. 5173.

2. D. Greenspan, "Supercomputer simulation of colliding microdrops of water," Computers Math. Applic., 19, 1990, p. 91.

3. D. Greenspan and L. F. Heath, "Supercomputer simulation of the modes of colliding microdrops of water," TR 273, Math. Dept., University of Texas at Arlington, 1990.

4. J. O. Hirschfelder, C. F. Curtiss and R. B. Bird, Molecular Theory of Gases and Liquids, Wiley, N.Y., 1967, p. 455.

5. I. Peterson, "Raindrop oscillation," Sci. News, 2, 1985, p.136.

6. S. F. Simpson and F. J. Haller, "Effects of experimental variables on mixing of solutions by collision of microdroplets," Analyt. Chem., 60, 1988, p. 2483. 


\section{APPENDIX A}

\section{FORTRAN PROGRAM DROP.FOR}

C TOTAL. NUMBER OF FAFTTCLES TS 41.02.

c. F AND $Q$ AFE MOLECULAR FAFAMETEFS 3 AND

OIMENSTON $X 0(4102)$, YO (41.23) , UXO (4102), UYO (4102),

$I X(4102,3), Y(4102,3), U X(4102,2), U Y(4102,2)$,

LACX(4102), ACY (4102) y $2(4102)$, FA(A102) , FX(4102),FY(4102), F(4102)

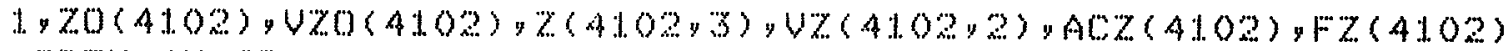

OFI:N (UNIT $=21$ )

OFEN (UNTY: 31 )

OFi:N (UNTY $: 41)$

$K=1$

KFFINT $=5000$

Do $4 \quad \mathrm{~T}=1,4102$

$X(I$, y l. $)=0$

$Y(T, y)=0$

$z(\mathrm{I}, \mathrm{t})=0$

UX(I, 1$)=0$

UY( $\mathrm{X}, 1)=0$

Uz $(\mathrm{x}, \mathrm{f})=0$

$X(1,2)=0 \%$

$Y(\pi, 2)=0$

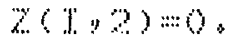

UX(1.?2) $=0$.

UY (T.2) $=0$

UZ( 1,2$)=0$

A(CX) II) $=0$

ACY $(T)=0$

$A(C), f)=0$.

CONT NUE:

READ $(21,10)(X O(T), Y O(T), Z O(I), U X O(T), U Y O(T), V Z O(I), I=1,4102)$

FORMAT (6F16.10)

$26 \%$ FOFMAT (T12, 20.5 )

(10) 30 II $=1.4 .02$

$X(T, I):=X O(T)$

$Y(I, 1)=Y O(I)$

$Z(I, I)=Z O(I)$

$U X(I, I)=U \times O(I)$

$U Y(I, I)=U Y O(I)$

$U Z(I, I)=U Z O(I)$

CONTTNUE

(30) TO 3456

100 $70 \quad I=1,4102$

$X(T, I)=X(T, 2)$

$Y(T, 1)=Y(T, Z)$

$Z(T, 4)=\cdots(T, z)$

$U X(I, I)=\because U X(I, 2)$

UY $(\mathrm{I}, \mathrm{I})=: \cup Y(\mathrm{X}, 2)$

$U z(x+1)=\cdots Z(x+2)$

70 ConTrMuE

$00 \%$ T.:1. 9102

$A C X(I)=0$.

ACY (T)

Al: $\%(\mathrm{~T})=0,0$

$F(x)=0$.

CONT INUE: 
3456

(10) 78 1. $=1,4.0 \%$

$A C X T=A B \times(T)$

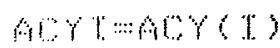

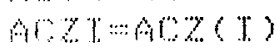

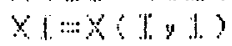

$Y(\cdots, Y(X) !)$

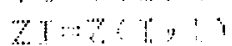

$\mathrm{T} F+1=1$

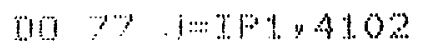

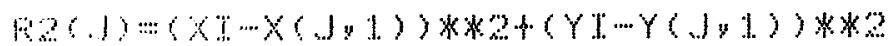

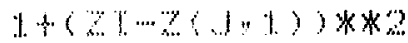

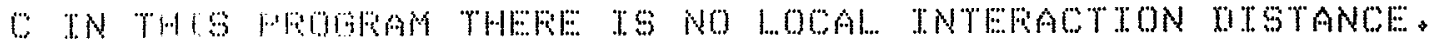

$F(1)=(-1.6,3+1.58,6 /(22(1)))$

i. $\quad(\log (1) \times 2(1))$

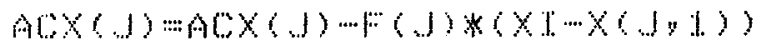

$\operatorname{ACY}(J) \cdots A C Y(J) \cdots \cdots(J) *(Y T \cdots Y(J y))$

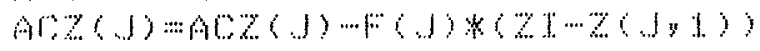

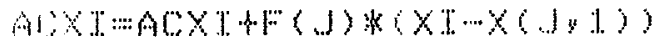

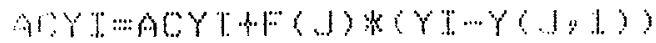

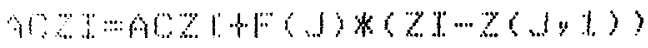

79 BNTINUE:

$A C \times(T) \cdots A C X I$

ACY (T) : ACYT

AC\% $(\mathrm{T}) \cdots A \mathrm{AOC}$

79 contronut:

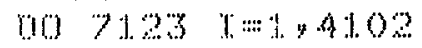

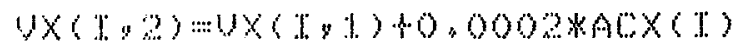

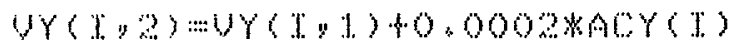

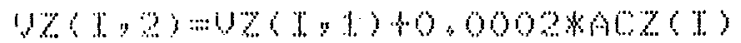

$X(1,2)=x(T ; 1)+0.0002 \times 0 \times(1,2)$

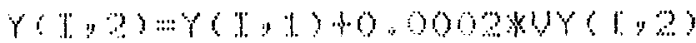

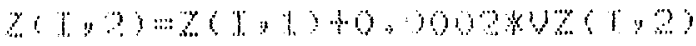

7123 CONTHNUI:

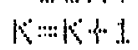

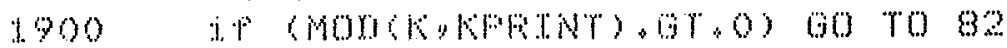

$x \in B E=0.0$

min $631 \%, 1,410 \%$

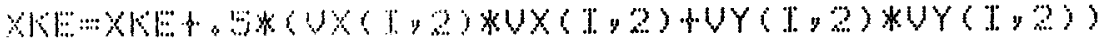

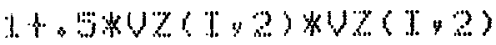

G\%: OONTINU:

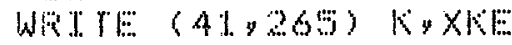

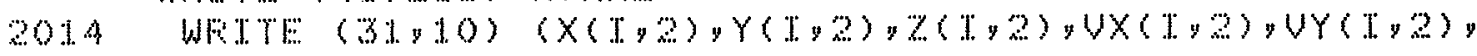

$1.22(T, 2), \pi=1,4102)$

a.

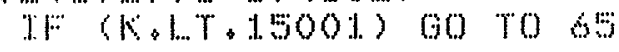

sTOF

INN 


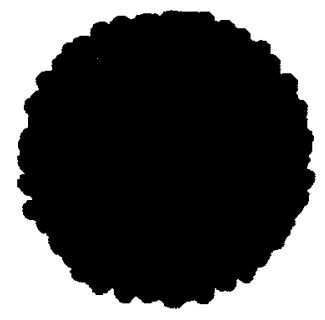

Figure 1. A microdrop of water at $45^{\circ} \mathrm{C}$.

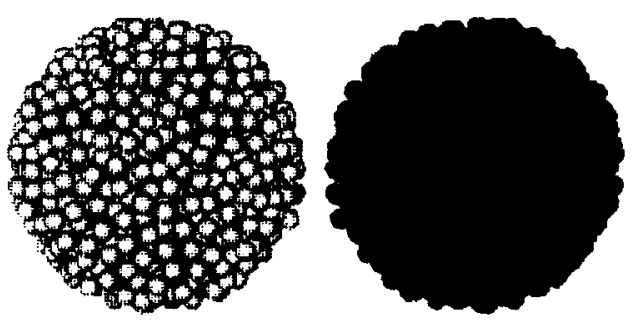

Figure 2. Two microdrops of water 3 \& apart. 

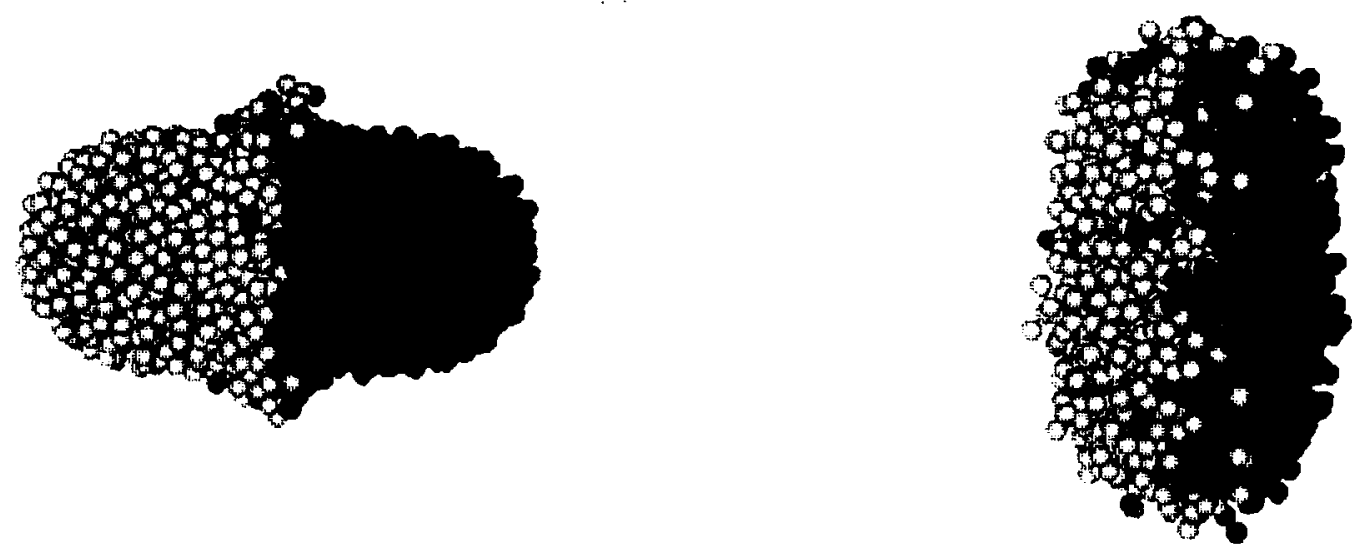

(a) $T_{9500}$

(b) $T_{18500}$
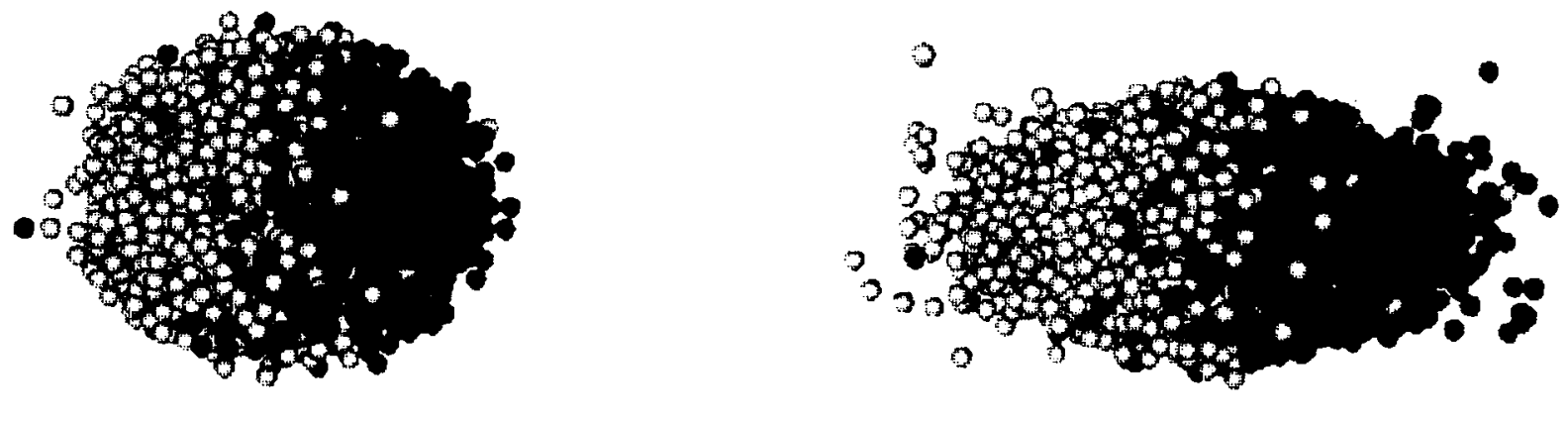

(c) $T_{27500}$

(d) $T_{36500}$

Figure 3. Oscillating oblateness mode. 


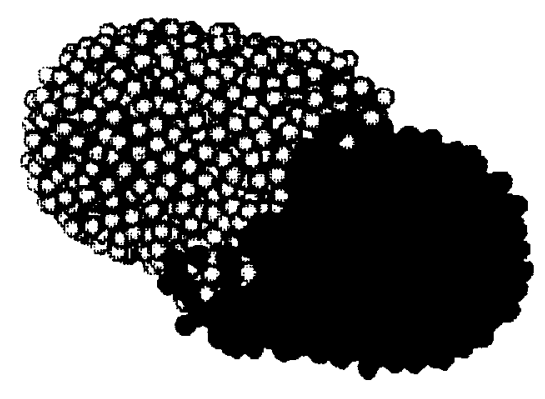

(a) $T_{9500}$

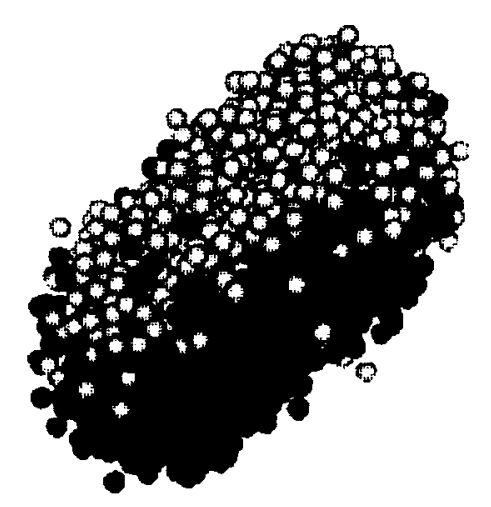

(b) $T_{18500}$

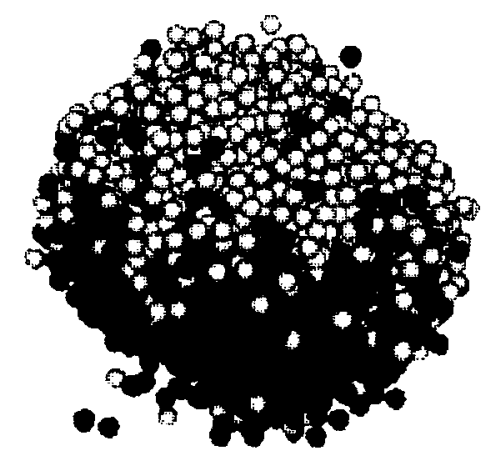
(c) $T_{27500}$

Figure 4. Raindrop mode. 

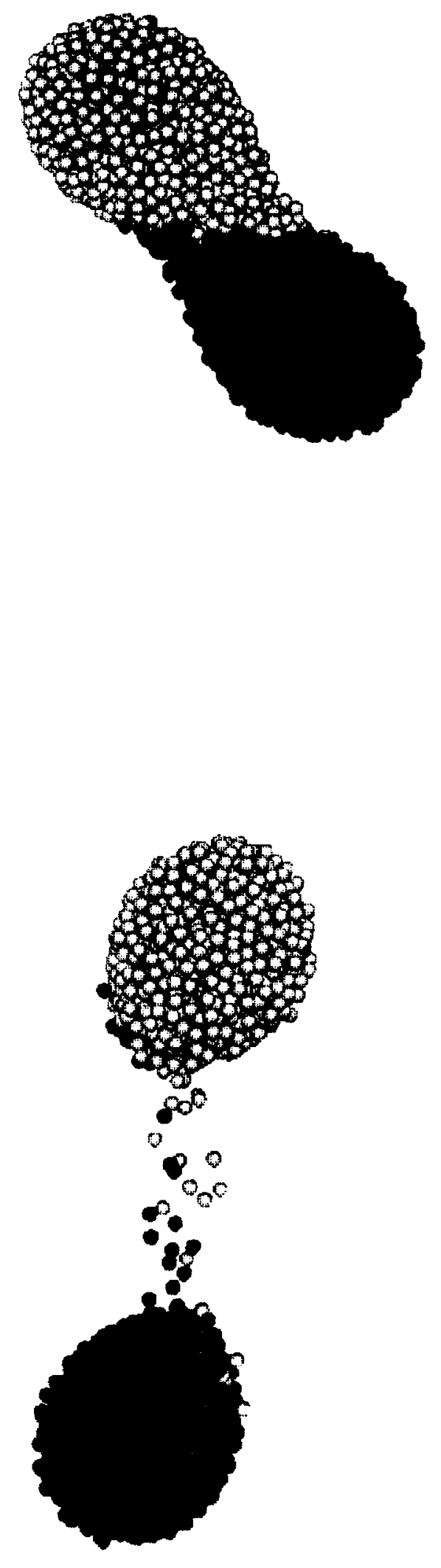

Figure 5.

Dumbell mode.
Figure 6. Brush

type collision

with teardrop modes. 


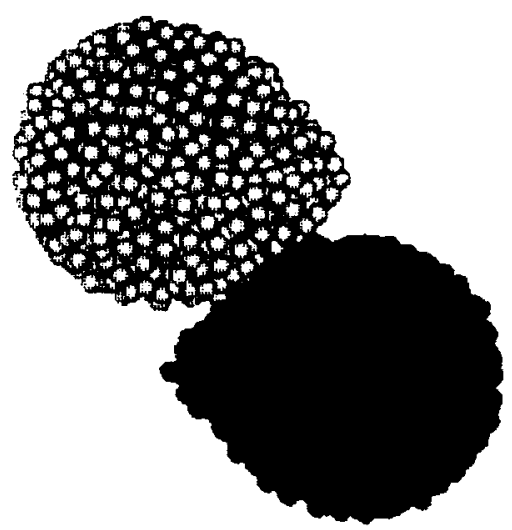

(a) $T_{4000}$

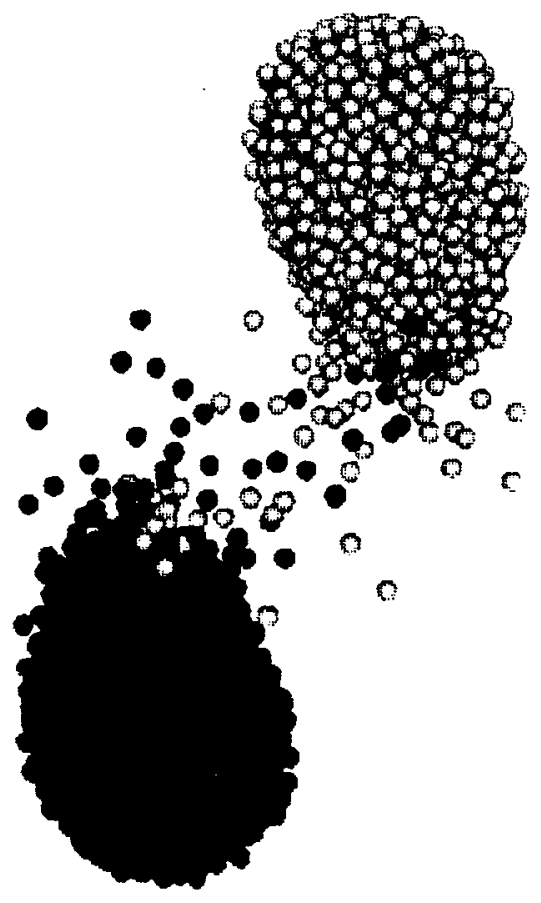

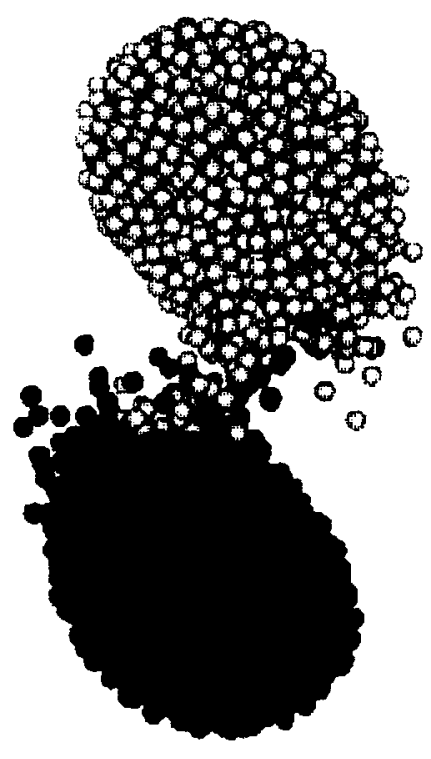

(b) $T_{9000}$

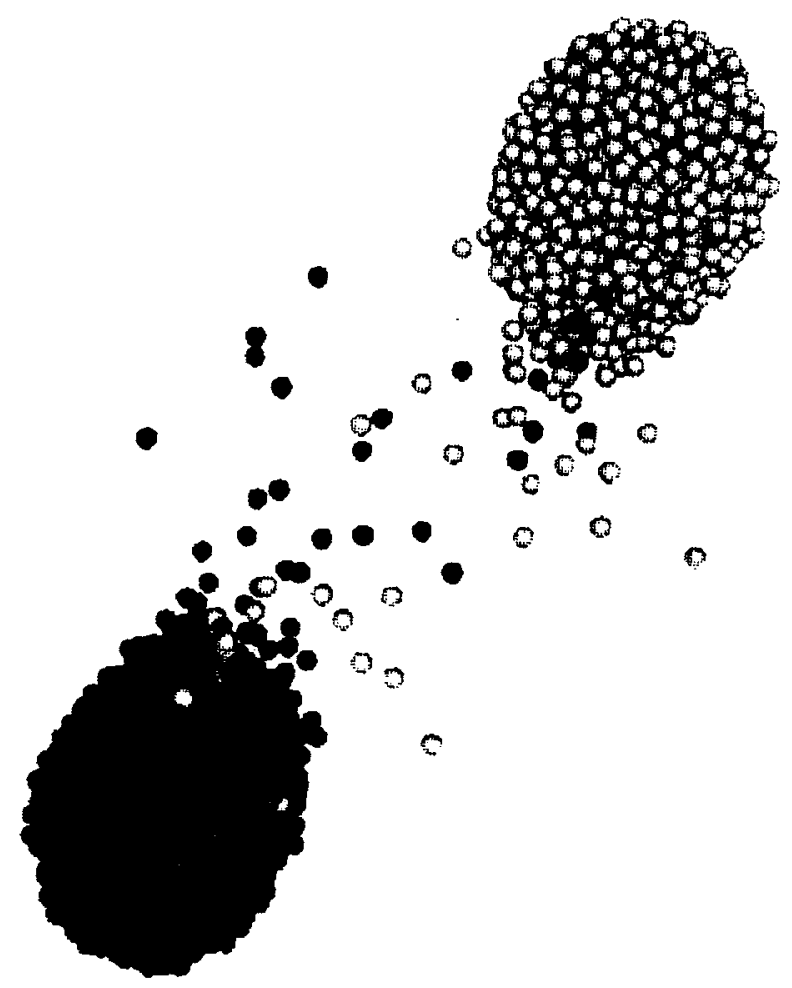

(c) $T_{13500}$

(d) $T_{18000}$

Figure 7. Soft collision. 


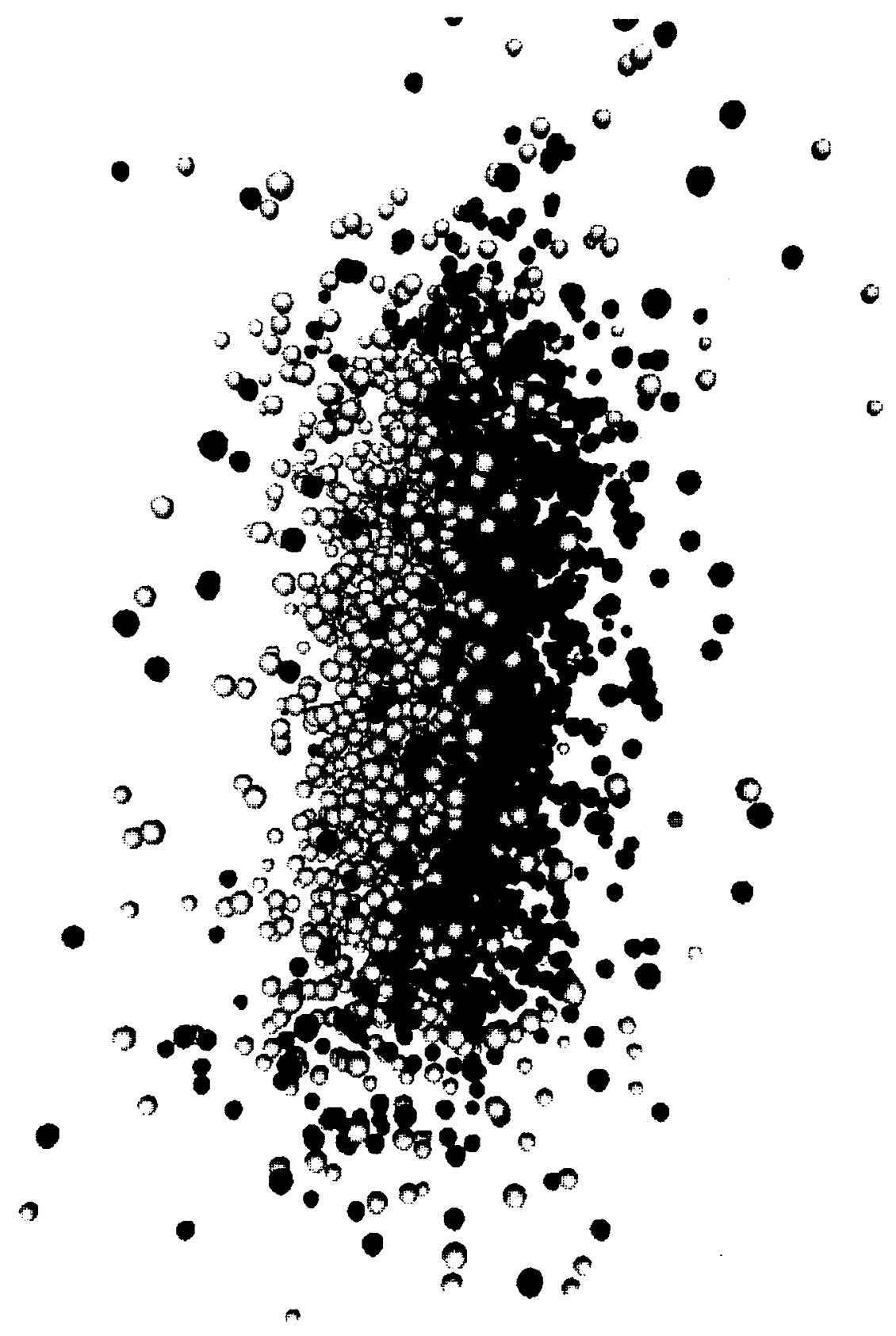

Figure 8. Direct, hard collision. 


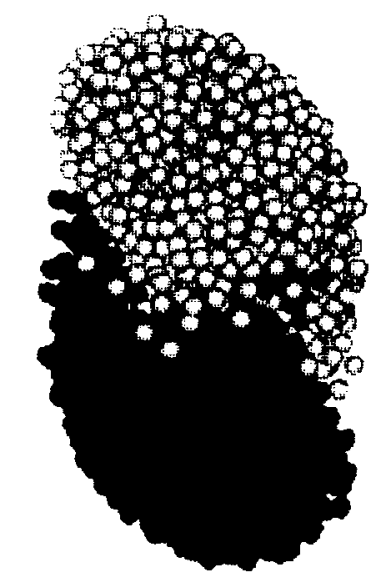

Figure 9. Peanut mode. 